\title{
Controlling crystallographic ordering in Mo-Cr-Ti-Al high entropy alloys to enhance ductility
}

Stephan Laube ${ }^{\mathrm{a}}$, Hans Chen ${ }^{\mathrm{a}}$, Alexander Kauffmann ${ }^{\mathrm{a},{ }^{*}}$, Steven Schellert ${ }^{\mathrm{b}}$, Franz Müller ${ }^{\mathrm{b}}$, Bronislava Gorr $^{\mathrm{b}}$, Julian Müller ${ }^{\mathrm{b}}$, Benjamin Butz ${ }^{\mathrm{b}}$, Hans-Juergen Christ ${ }^{\mathrm{b}}$, and Martin Heilmaier ${ }^{\mathrm{a}}$

${ }^{a}$ Institute for Applied Materials (IAM-WK), Karlsruhe Institute of Technology (KIT), EngelbertArnold-Str. 4, 76131 Karlsruhe, Germany

${ }^{\mathrm{b}}$ Institut für Werkstofftechnik, Universität Siegen, Paul -Bonatz-Str. 9-11, 57068 Siegen, Germany

* corresponding author

mail: alexander.kauffmann@kit.edu (A. Kauffmann)

phone: +4972160842346

\section{Highlights (3 to 5 Bullet points, max. 85 Characters per Bullet point)}

- Disordered A2 crystal structures are obtained in MoCrTi- $x \mathrm{Al}(\mathrm{x}=3,5$ at $\%)$

- The A2 crystal structure leads to higher ductility compared to B2 ordered alloys

- Appearance of order is appropriately described by thermodynamic calculations

- PLC effect is detected in all investigated compositions at elevated temperatures

\section{Abstract (193 / 250 words)}

Refractory high entropy alloys (RHEAs) from the Mo-Cr-Ti-Al system exhibit promising strength at elevated temperatures. However, at room temperature they possess a B2-ordered crystal structure and, therefore, exhibit reduced ductility. Guided by thermodynamic calculations, the Al concentration was systematically reduced in MoCrTi- $x \mathrm{Al}(x=25,15,10,5,3$ at $\%)$ in order to achieve a disordered, solid solution with A2-type crystal structure even at room temperature. The alloys were manufactured by arc melting followed by a homogenization treatment. To evaluate the chemical homogeneity, backscatter electron (BSE) imaging and energy dispersive X-ray spectroscopy (EDS) were performed. Dynamic differential scanning calorimetry (DSC) and selected area diffraction utilizing transmission electron microscopy (TEM-SAD) were performed for the: (i) detection of the characteristic heat signatures of the disorder-order transformations and (ii) determination of the ordering state at room temperature of the synthesized alloys, respectively. In case of MoCrTi-3A1, a single-phase A2 crystal structure without indications of a disorder-order transformation was obtained. Compression tests at temperatures ranging from room temperature up to $800^{\circ} \mathrm{C}$ reveal a significant improvement of plastic deformability at room temperature as compared to the formerly investigated equiatomic MoCrTiAl with its B2 crystal structure. 


\section{Keywords (6 / 6 words)}

high entropy alloys (HEA); crystal structure; calorimetry; mechanical properties; ductility; transmission electron microscopy (TEM)

\section{$1 \quad 1$ Introduction}

2 Based on the seminal work by Yeh et al. [1], the configurational entropy was considered to be a 3 predominant factor in stabilizing a solid solution in alloy systems with multiple principal elements (typically five or more). This simple guiding rule was assumed more important than the enthalpies of compound formation. According to this, multiple principal element, single-phase alloys with equiatomic or near equiatomic composition and five or more elements are called High Entropy Alloys (HEA) [1]. However, further research revealed that only few selected HEAs exhibit a chemically disordered solid solution with simple crystal structure in restricted temperature ranges [2-5]. Research in the field of Refractory High Entropy Alloys (RHEAs) with a body-centered cubic (BCC) crystal structure led to the development of some promising alloy compositions which exhibit extraordinary high-temperature strength in comparison to the commonly used Ni-based superalloys [5-12]. For example, the two equiatomic alloys MoNbTaW and MoNbTaVW exhibit an exceptionally high strength at temperatures up to $1600^{\circ} \mathrm{C}[6,8,13]$ and were reported to possess a single-phase, BCC crystal structure (Strukturbericht A2, W prototype). However, significant ductility is only observed at temperatures above $600^{\circ} \mathrm{C}$, which was calculated to be related to ordering in this system [14,15]. Further research with the objective of enhancing the specific mechanical properties led to the substitution of $\mathrm{W}, \mathrm{Mo}, \mathrm{Ta}$ with lighter elements like Ti, Hf and Zr. In contrast to the originally investigated alloys, this second generation of RHEAs suffers from lower, absolute yield stresses at elevated temperatures. They exhibit a reduced density of about $10 \mathrm{~g} / \mathrm{cm}^{3}[9,16,17]$. By the addition of $\mathrm{Al}$, several beneficial effects were achieved in comparison to the Al-free RHEAs, such as higher strength at RT and elevated temperatures. This may be due to the significantly different atomic radius of $\mathrm{Al}$ as compared to the other alloy elements, thus, resulting in strong solid solution strengthening of the material [18]. One remarkable finding was that the Al-containing RHEAs still possess a disordered solid solution, even though $\mathrm{Al}$ is known to form intermetallic phases with $\mathrm{Mo}$ or $\mathrm{Ti}$ as the other constituent elements of the examined alloys [19,20]. Furthermore, Al is known to promote the formation of B2 crystal structures as in NiAl, $\mathrm{FeAl}$ [21] or in HEAs like CrFeCoNi- $x \mathrm{Al}$ [22].

In contrast to face-centered cubic (FCC) metals and alloys, BCC metals and substitutional solid solutions feature a complex deformation behavior that depends on multiple parameters like temperature, strain rate and types of active slip systems. The dependence of BCC metals and dilute solid solutions can be roughly split into three temperature regimes [18,23-25]: First, below a critical temperature (typically $0.15-0.2 \cdot \mathrm{T}_{S}$ (with $\mathrm{T}_{S}$ being the solidus temperature), the plastic behavior is strongly influenced by the temperature and strain rate. This dependence results from non-planar cores of $\frac{a}{2}\langle 111\rangle$ screw dislocations (with $a$ being the lattice parameter). Calculations indicate that the core is extended with a three-fold symmetry in absence of an applied shear stress. In this temperature range, the motion of screw dislocations is thermally activated by kink pair nucleation. The kink pairs can subsequently propagate along the dislocation line when an external shear stress is applied. Second, above the critical temperature and below about $0.4 \cdot \mathrm{T}_{s}$, plasticity is mediated by the motion of screw and edge dislocations. In this temperature regime, the yield stress is almost temperature independent and strain-rate insensitive. Third, at temperatures above $0.4 \cdot \mathrm{T}_{s}$, the increasing impact of diffusional processes lead to a strain-rate 
dependence of the yield stress and a typical softening of the material is observed. However, in case of concentrated solutions some additional effects have to be considered like pinning mechanisms due to fluctuations of local composition or the formation of superjogs [26-28] as well as a transition from screw to edge dislocation dominated plastic yielding [29].

Besides the aforementioned complex temperature-deformation relationship, there is a transition from macroscopically ductile behavior to brittle fracture with decreasing temperature observed. This is influenced by the dislocation interaction near the crack tip [30]. The brittle-to-ductile transition arises due to the competition between of dislocation nucleation and propagation with the crack tip propagation. In case of a comparably simple metal, like pure $\mathrm{W}$, a correlation between grain size, dislocation density, crystallographic texture and the ductile to brittle transition could be established [31]. However, a model to describe this phenomenon in concentrated solid solutions has not been proposed or verified so far.

In the present work, we studied the microstructure and mechanical properties focusing on the ability for plastic deformation from RT up to $800^{\circ} \mathrm{C}$ in a series of Mo-Cr-Ti-Al alloys. The solute concentration of $\mathrm{Al}$ was systematically reduced from the well-characterized equiatomic quaternary $25 \mathrm{Mo}-25 \mathrm{Cr}-25 \mathrm{Ti}-$ $25 \mathrm{Al}[18,32,33]$ down to the non-equiatomic $32.3 \mathrm{Mo}-32.3 \mathrm{Cr}-32.3 \mathrm{Ti}-3 \mathrm{Al}$ (in at $\%$ ). The concentrations of the other alloying elements $\mathrm{Mo}, \mathrm{Cr}$ and $\mathrm{Ti}$ were kept at a constant ratio. The influence of $\mathrm{Al}$ on the deformation behavior is evaluated in this alloy series with the observed (and expected) significant enhancement of relative ductility at room temperature in the Al-lean alloys being rationalized based on the varying extents of crystallographic order.

\section{Experimental and materials}

\subsection{Manufacturing}

High purity bulk elements were used to manufacture alloy ingots by arc melting. The elements $\mathrm{Mo}, \mathrm{Cr}$, $\mathrm{Ti}$ and $\mathrm{Al}$ had nominal purities of 99.95, 99.5, 99.8 and $99.9 \%$, respectively, and were provided by chemPUR GmbH (Germany). Arc melting was conducted in an AM/0.5 furnace provided by Edmund Bühler GmbH (Germany). The furnace chamber was evacuated to $5 \cdot 10^{-2}$ mbar and filled with Ar. This process was repeated for three times in total to purify the melting atmosphere. Then a high vacuum of less than $1 \cdot 10^{-4}$ mbar was established. The processing chamber was then filled with Ar once more to a pressure of 600 mbar. Residual oxygen within the furnace chamber was gettered by liquefying a $\mathrm{Zr}$ globule before melting the bulk elements. Every manufactured ingot was flipped and re-melted at least five times before casting into a water-cooled $\mathrm{Cu}$ mold in a cuboid-shape.

The alloy ingots were homogenized utilizing a HTRH 70-600/18 resistance tube furnace by Carbolite Gero GmbH \& Co. KG (Germany) with flowing high purity Ar atmosphere. Depending on the alloy composition, the heat treatment temperatures were 1200 and $1500^{\circ} \mathrm{C}$, respectively, for a dwell time of $20 \mathrm{~h}$. Heating and cooling was conducted at a rate of $250 \mathrm{~K} / \mathrm{h}$.

\subsection{Thermodynamic characterization and thermal analysis}

Thermodynamic calculations were performed utilizing FactSage V7.2 in combination with an in-house database. The calculations were carried out at a step size of $100 \mathrm{~K}$.

High temperature differential scanning calorimetry (DSC) experiments were conducted in a DSC 404 F1 Pegasus ${ }^{\circledR}$ equipped with a platinum furnace supplied by Netzsch-Gerätebau GmbH (Germany). A sapphire standard was used as reference sample to determine the derivative of enthalpy with respect to temperature $d H / d T$ according to DIN 51007. The DSC chamber was evacuated and flushed with Ar three times before a constant and controlled high purity Ar flow was established. In order to remove 
traces of residual oxygen in the gas atmosphere, a $\mathrm{Zr}$ getter was mounted on the sample carrier. The DSC samples were placed in Pt-Rh crucibles with $\mathrm{Al}_{2} \mathrm{O}_{3}$ inlets and were covered by a Pt-Rh lid. All experiments were performed at a heating rate of $20 \mathrm{~K} / \mathrm{min}$ at temperatures ranging from RT up to $1450^{\circ} \mathrm{C}$

\subsection{Sample preparation and characterization}

The composition of the homogenized equiatomic MoCrTiAl alloy was determined by means of inductively coupled plasma-optical emission spectrometry (ICP-OES). Helium carrier gas hot extraction was utilized to determine the impurity level of $\mathrm{O}$ and $\mathrm{N}$ using at least three samples of every composition using a TC500 system provided by Leco Instrumente GmbH (Germany).

Samples for microstructural investigations were prepared by water-cooled grinding with $\mathrm{SiC}$ paper followed by standard metallographic polishing steps with $3 \mu \mathrm{m}$ and $1 \mu \mathrm{m}$ polycrystalline diamond suspensions. After polishing, the samples were etched for 5 min using a reagent consisting of $\mathrm{NH}_{3}, \mathrm{H}_{2} \mathrm{O}_{2}$ and distilled water in a 1:2:5 ratio. Subsequently, a chemo-mechanical vibratory polishing step was conducted for at least $8 \mathrm{~h}$. A non-crystallizing oxide polishing suspension with $\mathrm{pH}=9.8$ provided by Struers (Germany) was utilized. Powder samples for X-ray diffraction (XRD) were obtained by crushing bulk materials in an agate mortar. XRD was performed using a Bruker D2 Phaser (Germany) equipped with a 1D LynxEye detector. The $\mathrm{Cu} K \alpha$ source was operated at $30 \mathrm{kV}$ and $10 \mathrm{~mA}$. $2 \Theta$ was scanned from 10 up to $145^{\circ}$ at a step size of $0.01^{\circ}$ in $\Theta-\Theta$ geometry at an accumulated acquisition time of $384 \mathrm{~s}$ per step. The lattice parameter of the cubic crystal structure $a$ is determined using extrapolation of the peak positions to $\Theta=90^{\circ}$ utilizing the weighted function $w(\Theta)=1 / 2 \cdot\left(\cot ^{2} \Theta+\cot \Theta \cdot \cos \Theta\right)$ in order to minimize the influence of systematic errors [34].

Additional diffraction experiments were performed by means of Selected Area Diffraction (SAD) utilizing a Transmission Electron Microscope (TEM). The Talos F200X supplied by FEI (USA) operated at an acceleration voltage of $200 \mathrm{kV}$. Thin foils for TEM analysis were prepared from bulk specimens. The platelets were cut by diamond wire saw and then mechanically ground with SiC paper to a thickness of about $100 \mu \mathrm{m}$. For the final thinning, the foils were jet-polished with a TenuPol-5 provided by Struers $\mathrm{GmbH}$ at $295 \mathrm{~K}$ at a voltage of $12 \mathrm{~V}$ until perforation. The electrolyte was composed of $\mathrm{H}_{2} \mathrm{SO}_{4}$ and $\mathrm{CH}_{3} \mathrm{OH}$ in a 4:1 ratio. The microstructure of the samples was analyzed by means of Scanning Electron Microscopy (SEM) using an EVO50 from Carl Zeiss AG (Germany) equipped with a Backscatter Electron (BSE) detector. Energy dispersive X-ray spectroscopy (EDS) was performed utilizing a silicon drift detector by Thermo Fisher Scientific Inc. (USA) at an acceleration voltage of $30 \mathrm{kV}$.

In order to characterize the compressive yield stress $\sigma_{0.2}$ and deformation behavior, quasi-static compression tests were carried out on a Z100 electro-mechanical universal testing machine supplied by ZwickRoell GmbH \& Co. KG (Germany). Compression test samples with (4 x 3 x 3) $\mathrm{mm}^{3}$ in size were extracted by electrical discharge machining, followed by a standard metallographic procedure in order to ensure co-planar, flat compression surfaces. Yield stress was determined at an off-set plastic strain of $0.2 \%$. The tests were performed at temperatures ranging from RT up to $800^{\circ} \mathrm{C}$ at an engineering strain rate of $10^{-3} \mathrm{~s}^{-1}$ with respect to the initial sample height. The strain was detected by strain gauges. To prevent any superimposed effect from oxidation at elevated temperatures, the tests were performed in a vacuum furnace supplied by Maytec $\mathrm{GmbH}$ (Germany). The tests were carried out at a pressure of about $1 \cdot 10^{-4} \mathrm{mbar}$. SiC punches were used and hexagonal BN was applied for lubrication. 
Table 1: Determined chemical composition $x$ of the investigated alloys, given in at $\%$. Inductively coupled plasma-optical emission spectrometry (ICP-OES) is indicated by * and standard-related energy dispersive X-ray spectroscopy (EDS) by $\uparrow$. O and $\mathrm{N}$ concentration where determined by means of carrier gas hot extraction and presented in wt.-ppm.

\begin{tabular}{|c|c|c|c|c|c|c|c|c|}
\hline \multirow[t]{2}{*}{ abbreviation } & \multirow{2}{*}{$\begin{array}{c}\text { heat } \\
\text { treatment }\end{array}$} & \multirow{2}{*}{ nominal composition / at $\%$} & \multicolumn{4}{|c|}{$\bar{x} / \mathbf{a t} \%$} & \multicolumn{2}{|c|}{$\bar{x} / \mathbf{p p m}$} \\
\hline & & & Mo & $\mathrm{Cr}$ & $\mathrm{Ti}$ & $\mathrm{Al}$ & $\mathrm{O}$ & $\mathrm{N}$ \\
\hline MoCrTiAl* & $1200^{\circ} \mathrm{C} / 20 \mathrm{~h}$ & $25 \mathrm{Mo}-25 \mathrm{Cr}-25 \mathrm{Ti}-25 \mathrm{Al}$ & 24.8 & 24.9 & 24.9 & 25.4 & $440 \pm 5$ & $<0.5$ \\
\hline MoCrTi-15Al $\dagger$ & $1200^{\circ} \mathrm{C} / 20 \mathrm{~h}$ & $28.33 \mathrm{Mo}-28.33 \mathrm{Cr}-28.33 \mathrm{Ti}-15 \mathrm{Al}$ & 29.5 & 27.7 & 27.8 & 15.0 & $231 \pm 50$ & $<0.5$ \\
\hline MoCrTi-10Al $\dagger$ & $1200^{\circ} \mathrm{C} / 20 \mathrm{~h}$ & 30Mo-30Cr-30Ti-10Al & 29.9 & 29.9 & 29.6 & 10.6 & $279 \pm 65$ & $<0.5$ \\
\hline MoCrTi-5Al $\dagger$ & $1500^{\circ} \mathrm{C} / 20 \mathrm{~h}$ & 31.66Mo-31.66Cr-31.66Ti-5Al & 32.4 & 31.3 & 31.1 & 5.2 & $291 \pm 72$ & $<0.5$ \\
\hline MoCrTi-3Al $\dagger$ & $1500^{\circ} \mathrm{C} / 20 \mathrm{~h}$ & 32.33Mo-32.33Cr-32.33Ti-3Al & 32.0 & 32.7 & 32.1 & 3.2 & $134 \pm 50$ & $<0.5$ \\
\hline
\end{tabular}

124

125

126

127

128

129

130

\section{Results and discussion}

Based on previous results of ordered alloys from the Mo-Cr-Ti-Al system [32], thermodynamic calculations were performed to assess the equilibrium phases in a temperature range between 800 and $1700{ }^{\circ} \mathrm{C}$. According to these calculations, a second-order phase transformation from A2 to B2 crystal structure occurs at about $1110^{\circ} \mathrm{C}$ for the equiatomic MoCrTiAl. B2 maintains the major thermodynamically stable crystal structure down to RT. However, a possible low phase fraction A15 $\left(\mathrm{Al}(\mathrm{Mo}, \mathrm{Nb})_{3}\right)$ appears below $850^{\circ} \mathrm{C}$ as well [32], although it was not found experimentally.

As depicted in Fig. 1a, by reducing the Al content the A2-B2 second-order phase transformation in MoCrTi-15Al occurs at about $1000^{\circ} \mathrm{C}$ and, therefore, about $100 \mathrm{~K}$ lower compared to the equiatomic alloy. Below about $800^{\circ} \mathrm{C}$, the precipitation of A15 is predicted similarly to the equiatomic alloy. Further calculations indicate that even lower $\mathrm{Al}$ concentrations lead to a decrease of the A2-B2 transformation temperature to below $800^{\circ} \mathrm{C}$ (see Fig. $1 \mathrm{~b}$ for MoCrTi-3Al). No A15 formation is predicted in these alloys with $\mathrm{Al}$ concentrations lower than $15 \mathrm{at} \%$. Note that the solidus temperature of the respective alloys increases with decreasing Al concentration; however, the absolute change is only about $100 \mathrm{~K}$ for the investigated composition range. This is of importance in the further course of the present article when the temperature-dependent mechanical properties of the alloys are assessed.
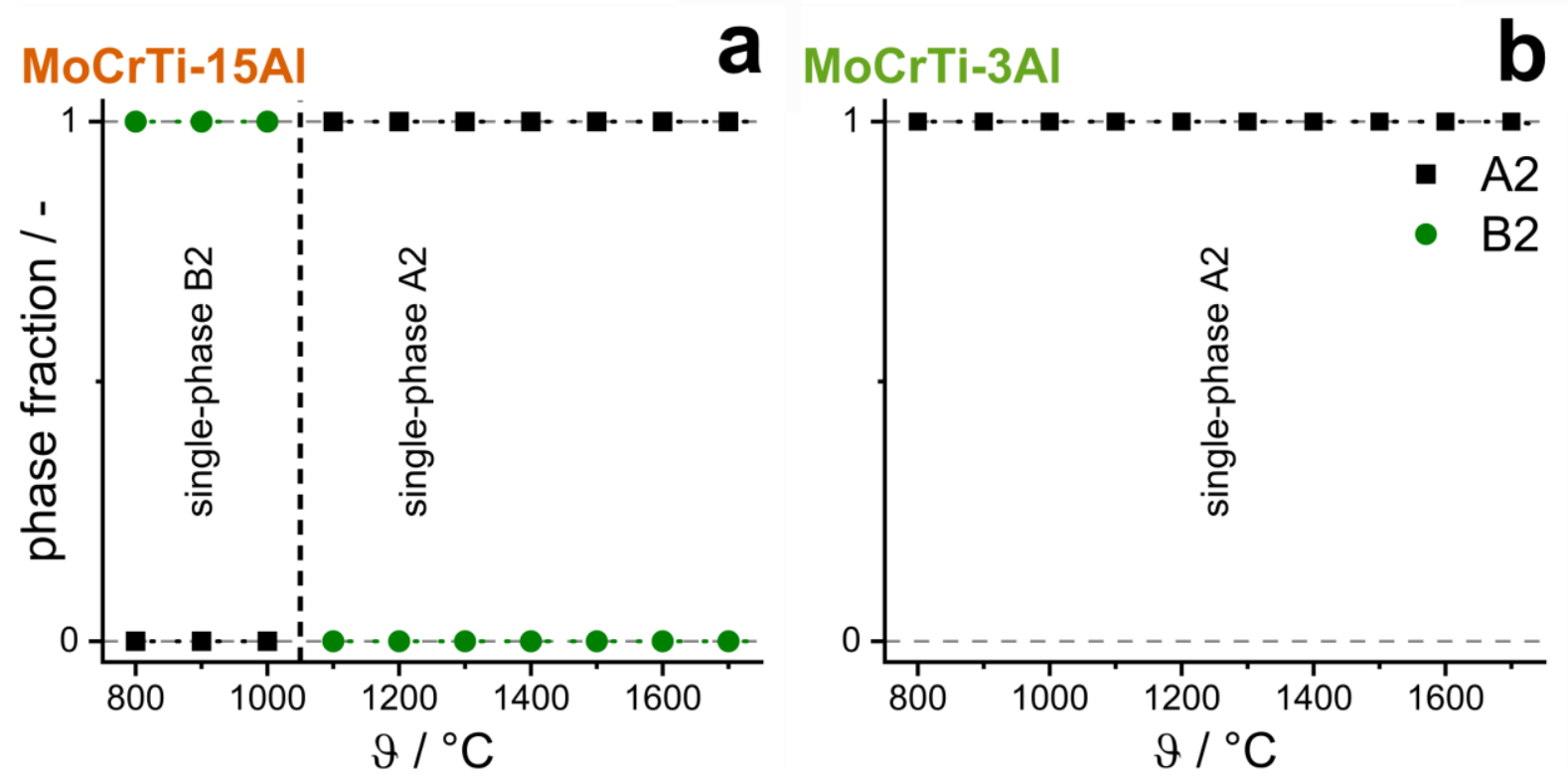

Fig. 1: Equilibrium phase fractions as functions of temperature $\vartheta$ as predicted by the thermodynamic calculations for: a) MoCrTi-15Al; b) MoCrTi-3Al. The step size of the calculations was $100 \mathrm{~K}$ (as highlighted by the respective closed symbols). 
In order to investigate the influence of Al-concentration over a broad composition range, MoCrTiAl, MoCrTi-15Al, MoCrTi-10Al, MoCrTi-5Al and MoCrTi-3Al were manufactured. The equiatomic MoCrTiAl alloy was chosen as reference alloy for this investigation due to its well-known properties $[18,32]$. Table 1 provides the chemical composition by means of ICP-OES and standard-related EDS of the other alloys in the as-homogenized state. The determined alloy compositions are in good agreement with the nominal compositions. Al evaporation during arc melting is not observed. Subsequent to the homogenization treatment, all investigated alloys exhibit a microstructure consisting of equiaxed grains, as displayed in Fig. 2a \& b. Secondary phase are not detected by means of SEM-BSE investigations within the grains or at the grain boundaries. However, all alloys exhibit some porosity by casting and the Kirkendall effect [35] (black features in Fig. 2a \& b) within the grains after the homogenization treatment. Due to the known significant influence of interstitial elements on the mechanical behavior, the $\mathrm{N}$ and $\mathrm{O}$ concentrations were determined (Table 1): average $\mathrm{O}$ contents of the non-equiatomic samples are below 300 wt.-ppm and the $\mathrm{N}$ concentrations are below the detection limit of $0.5 \mathrm{wt}$.-ppm. Hence, it can be assumed that there is no relevant impact on the mechanical behavior.

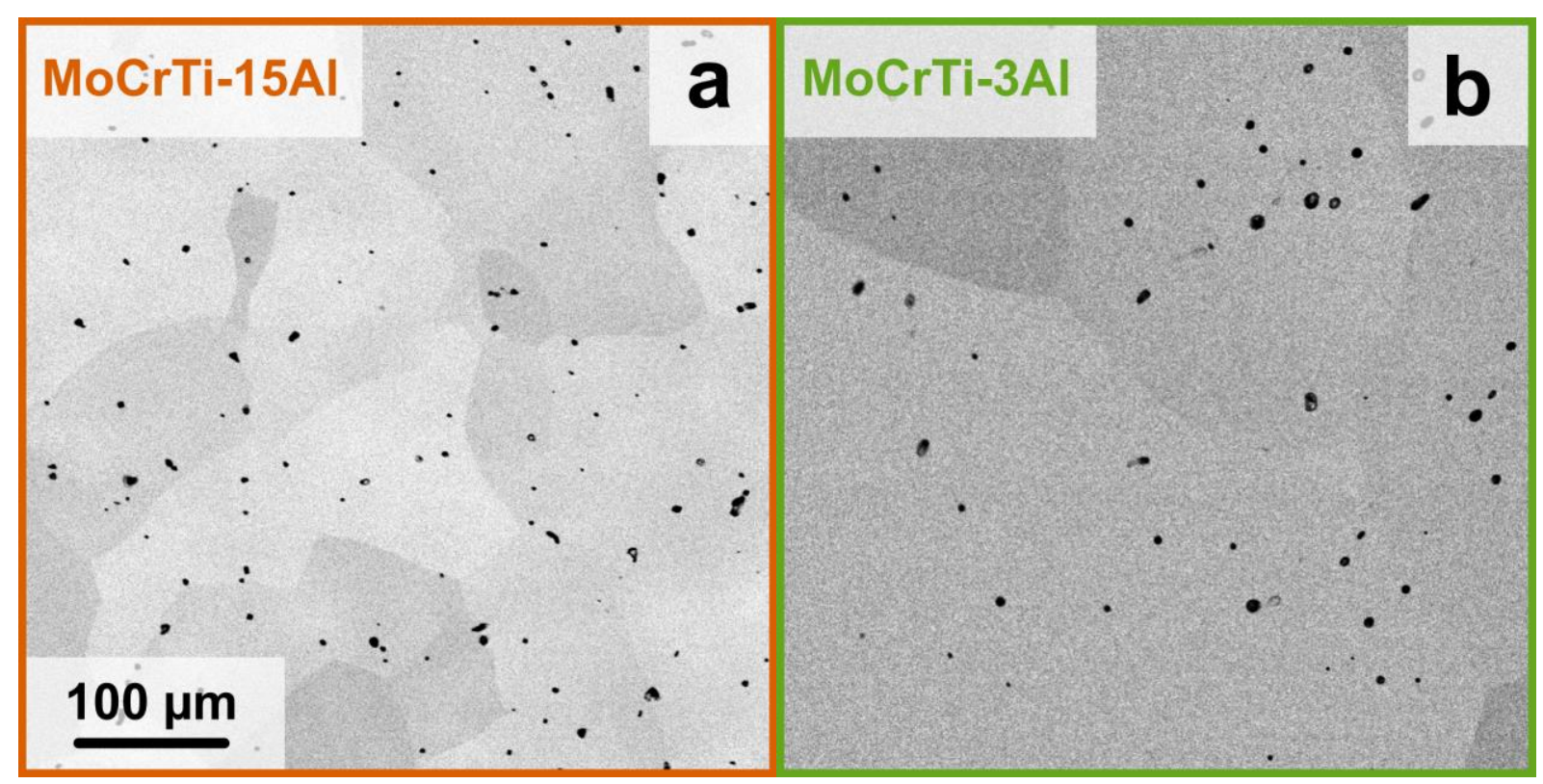

Fig. 2: SEM-BSE micrographs in the as-homogenized state of: a) MoCrTi-15Al; b) MoCrTi-3Al. Black features are pores related to the Kirkendall effect during homogenization treatment. Same magnification is used for both micrographs.

The diffraction patterns of the investigated alloys are depicted in Fig. 3 with a logarithmic scale for better visibility of possible low intensity Bragg peaks. The determined lattice parameters are 3.096(2), 3.095(8), 3.088(6) and 3.092(2) ̊̊ for MoCrTi-15Al, MoCrTi-10Al, MoCrTi-5Al and MoCrTi-3Al, respectively. For comparison, the lattice parameter of the equiatomic MoCrTiAl is 3.099(8) $\AA$ [18]. All detected Bragg peaks in MoCrTi-3Al, MoCrTi-5Al and MoCrTi-15Al can be assigned to an A2 crystal structure (space group $\operatorname{Im} \overline{3} \mathrm{~m}, \mathrm{~W}$ prototype). No indications for B2 superlattice peaks are found in these alloys. In contrast, the diffraction pattern of MoCrTi-10Al exhibits a Bragg peak that can be assigned to $\{100\}$-type lattice planes. This peak suggests the presence of $\mathrm{B} 2$ order (space group $\mathrm{Pm} \overline{3} \mathrm{~m}, \mathrm{CsCl}$ prototype). Additional B2 superlattice peaks apart from the $\{100\}$-peak (e.g. $\{111\},\{210\}, \ldots$ ) are not detected by means of X-ray diffraction.

For the present alloy series, a simultaneous change of site occupation in the B2 crystal structure and a change of the transformation temperatures to lower temperatures is expected. For the lowest $\mathrm{Al}$ contents, no ordering might be observed at all. As pointed out in Ref. [32], the specific site occupation in the 
equiatomic MoCrTiAl leads to vanishing Bragg intensity for the B2 superlattice peaks due to similar mean scattering cross sections for the respective lattice sites. While B2 order is likely in MoCrTi-15Al, the site occupations are obviously not sufficiently changed to induce significant intensity of superlattice peaks in XRD. For MoCrTi-10Al in contrast, a small $\{100\}$-peak is experimentally seen indicating that the site occupation is changed by the reduction of the Al content. Accordingly, $\mathrm{Al}$ is contributing to order. Nevertheless, the composition dependent site occupation factors in the alloys are still unclear, notwithstanding some partial order. For even lower Al contents, the A2-B2 transformation temperature might already be low enough to obtain A2 down to room temperature, which would be consistent with the absence of superlattice peaks in Fig. 3.

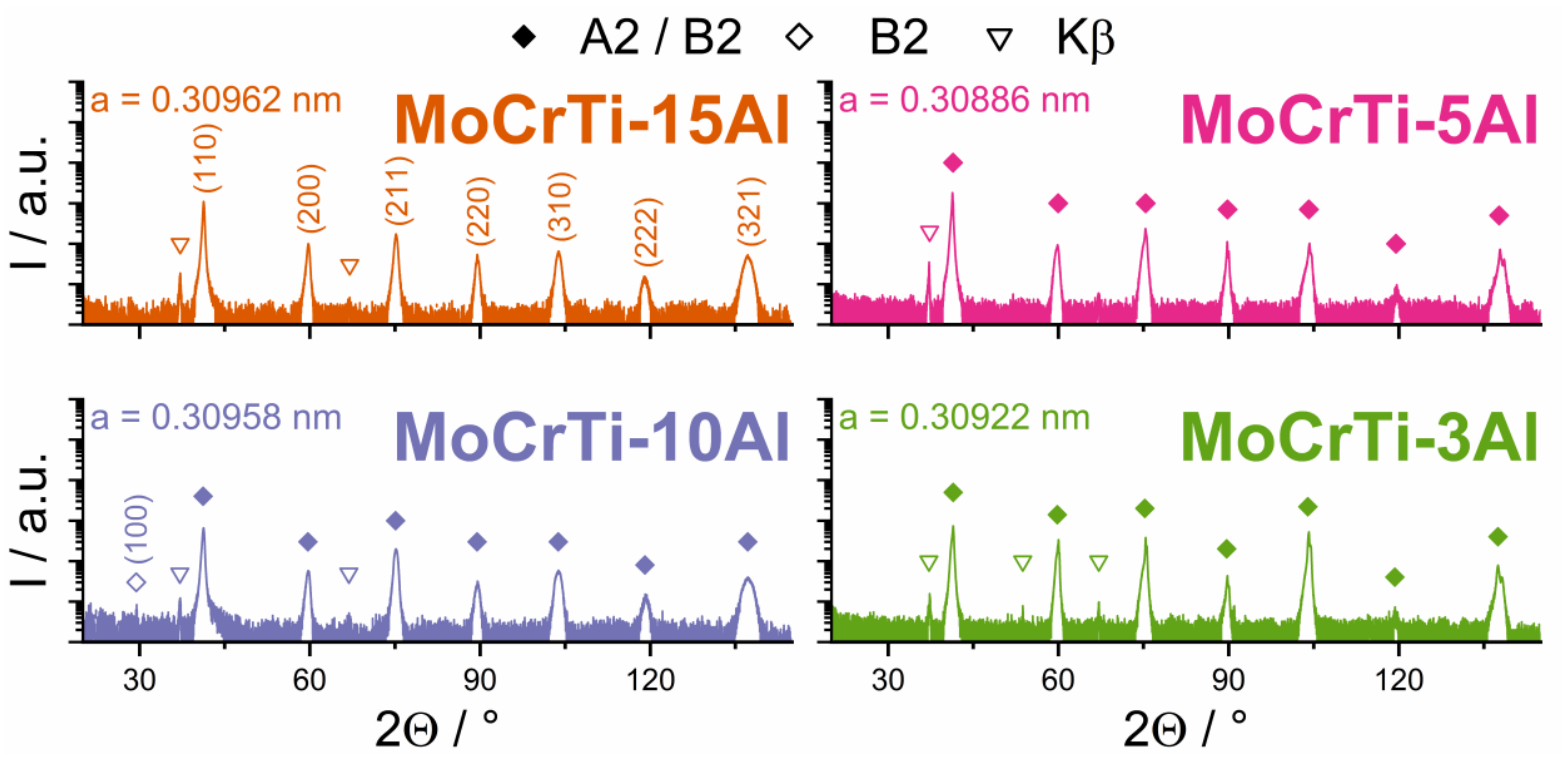

Fig. 3: Powder XRD patterns of the investigated alloys. Closed diamonds indicate A2 / B2 Bragg peaks, open diamonds highlight visible B2 Bragg peaks. The intensity scale is plotted in a logarithmic fashion for better visibility of low intensity peaks. Bragg peaks arising from residual $\mathrm{Cu} \mathrm{K} \beta$ radiation are indicated by open triangles.

176

To further investigate the ordering phenomena, electron diffraction was employed. Already in the case of the equimolar MoCrTiAl [32], B2 ordering was verified by TEM-SAD due to its higher dynamic range in comparison to XRD and, therefore, higher sensitivity for super lattice diffractions spots of low intensity. Therefore, TEM-SAD pattern close to [001] zone axes (ZA) for MoCrTi-15Al and MoCrTi$3 \mathrm{Al}$ were obtained. The patterns are displayed in Fig. 3a \& b, respectively. MoCrTi-15Al clearly exhibits characteristic B2 diffraction spots, while in MoCrTi-3Al no indications for ordered crystal structure are found. Additionally, the presence of antiphase domain boundaries (APDBs) in MoCrTi-15Al (not shown here) supports the beforehand calculated A2-to-B2 transformation. In agreement with the missing super lattice diffraction spots, no APDBs are found in MoCrTi-3Al. 


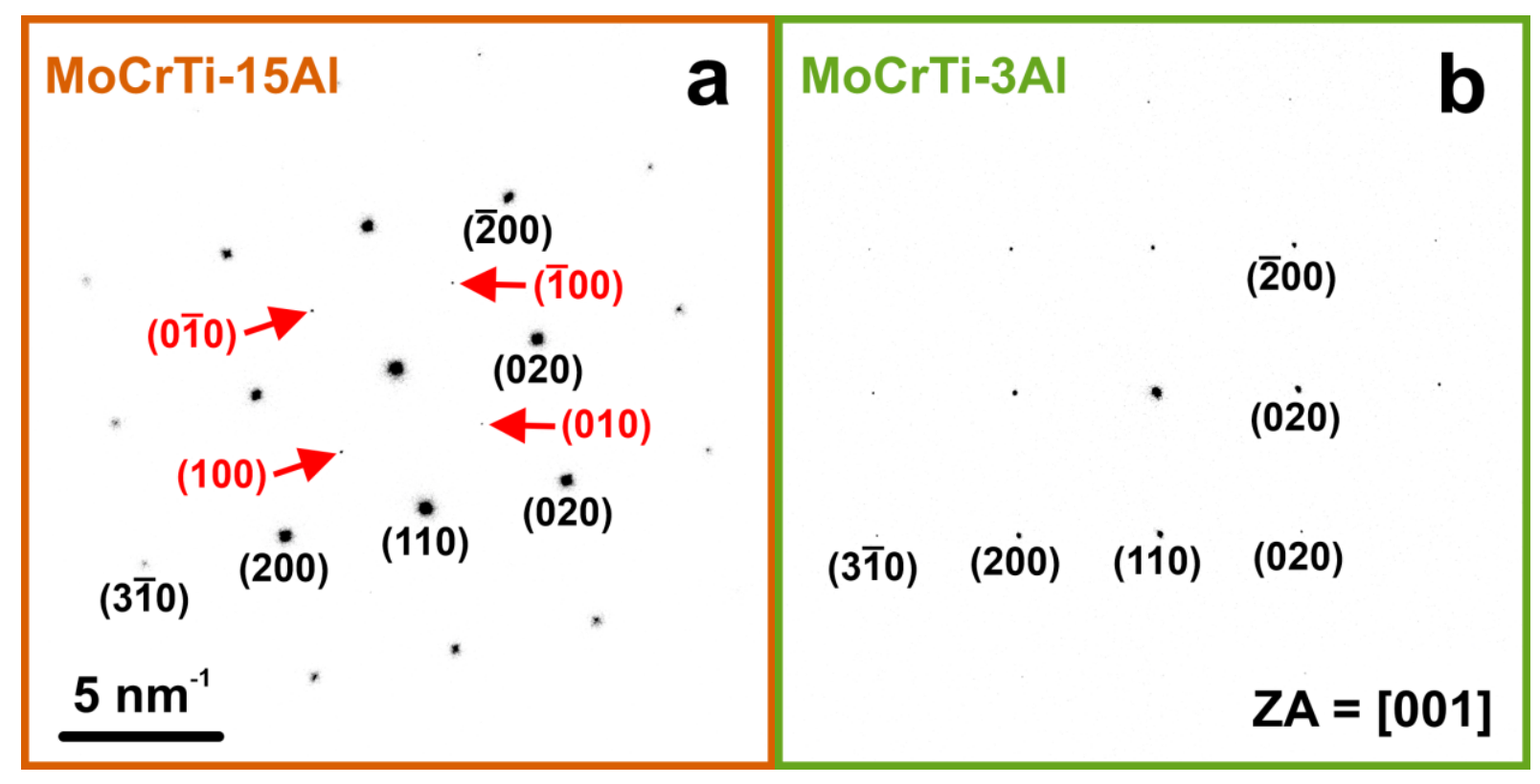

Fig. 4: TEM experiments. Selected Area Diffraction (SAD) patterns of: a) MoCrTi-15Al; b) MoCrTi-3Al. Superlattice spots of the B2 crystal structure are marked with red arrows and are labeled accordingly. For detailed description, the reader is referred to the text. Both pattern are acquired close to [001] ZA and with the same magnification.

Further investigations on the peculiarities of the ordering transformation were conducted by DSC experiments. The temperature dependence of the derivative of enthalpy with respect to temperature $d H / d T$ is presented in Fig. 5a for MoCrTi-15Al and MoCrTi-3Al (at a heating rate of $20 \mathrm{~K} / \mathrm{min}$ ). The equiatomic MoCrTiAl and also the MoCrTi-15Al and MoCrTi-10Al exhibit a $\lambda$-shaped peak. The peak temperatures are indicated by triangles in Fig. 5a. The $\lambda$-shaped peaks most likely indicate second-order or continuous phase transformation [36]. The transformation temperature $\left(T_{\mathrm{c}}\right)$ does not change by the variation of heating rate (not shown here) [32] and $d H / d T$ exhibits a power law behavior at $T_{\mathrm{c}}$ [37]. Therefore, a second-order phase transformation can be assigned to the crystallographic ordering from $\mathrm{A} 2$ to $\mathrm{B} 2$ and vice versa in these alloys. The thermodynamic calculations predict $T_{\mathrm{c}}$ at about 1110,1050 and $1000^{\circ} \mathrm{C}$ for $\mathrm{MoCrTiAl}$, MoCrTi-15Al and MoCrTi-10Al, respectively. The experimentally determined $T_{\mathrm{c}}$ decrease with decreasing $\mathrm{Al}$ concentration, from $T_{\mathrm{c}}=990^{\circ} \mathrm{C}$ in the case of the equiatomic MoCrTiAl to 965 and $860^{\circ} \mathrm{C}$ for MoCrTi-15Al and MoCrTi-10Al, respectively. The offset between the calculated and the experimental determined phase transition temperatures is consistently only about $100 \mathrm{~K}$. This difference might be addressed in further refinement steps of the thermodynamic database in future work. MoCrTiAl, MoCrTi-15Al and MoCrTi-10Al exhibit a $d H / d T$-plateau feature (temperature range is highlighted by the respective background color in Fig. 5a) between the onset temperature $T_{\text {onset }}$ and $T_{\mathrm{c}}$. $T_{\text {onset }}$ as a characteristic temperature is chosen because the plateau feature is heating rate dependent and, therefore, not of second-order type. Nevertheless, the microscopic origin of this plateau remains unknown. In Ref. [32], it is speculated to be related to diffusional wetting of the APDBs. For MoCrTi-5Al and MoCrTi-3Al $d H / d T$ does not exhibit a $\lambda$-shaped peak. Instead, a gentle broad Gaussian $d H / d T$-peak at $T^{*}$ is observed at 760 and $775^{\circ} \mathrm{C}$ (star-shaped symbol in Fig. 5a \& b). 

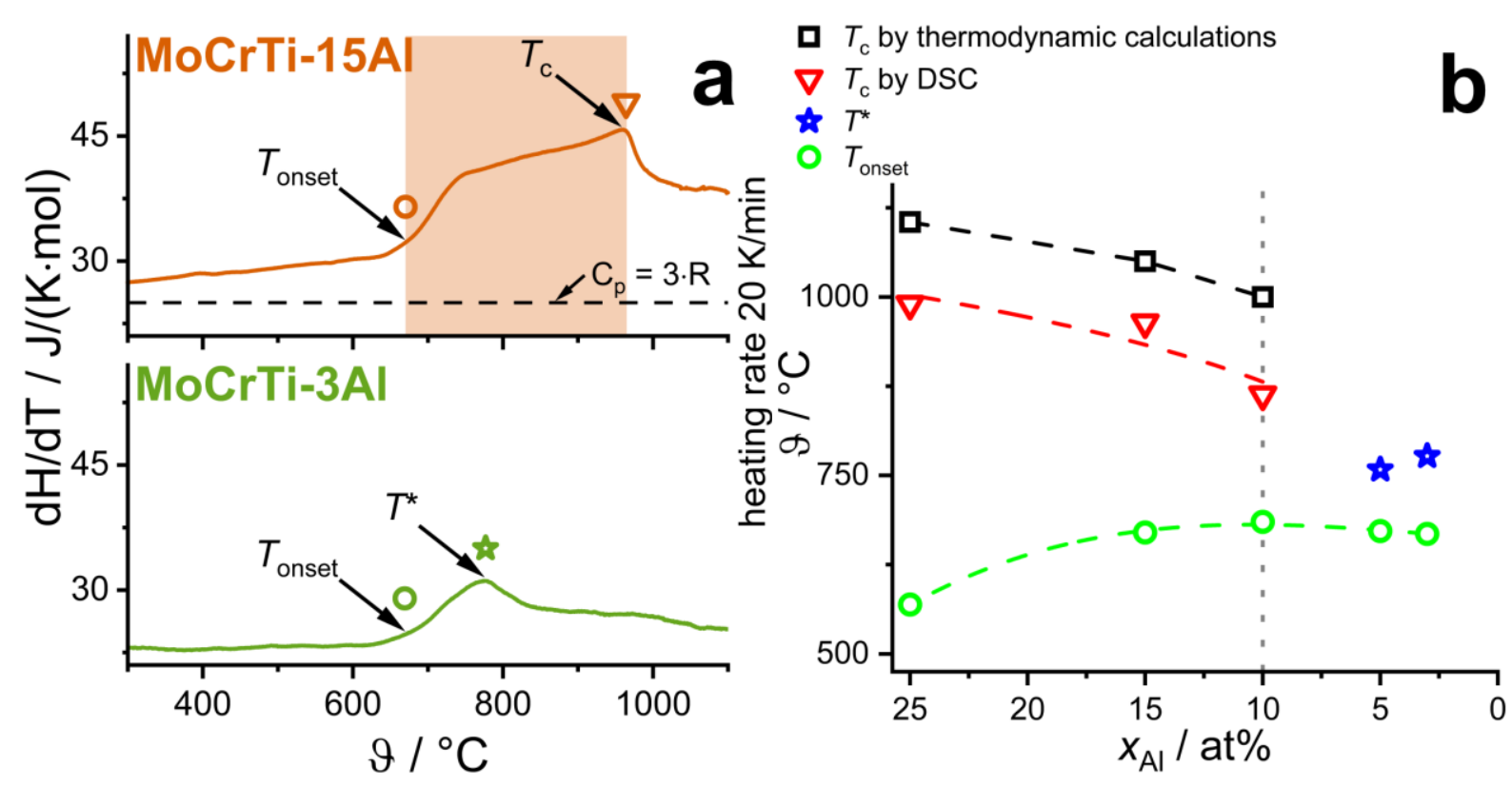

Fig. 5: a) Derivative of enthalpy with respect to temperature $d H / d T$ of MoCrTi-15Al (upper) and MoCrTi-3Al (lower). DSC was performed with a heating rate of $20 \mathrm{~K} / \mathrm{min}$. Open circles mark the onset temperature $T_{\text {onset }}$; star shaped symbols indicate the Gaussian $d H / d T$-peak temperature $T^{*}$ and the open triangles mark the transformation temperature $T_{\mathrm{c}}$. b) Summary of the predicted phase transformation temperatures and experimentally determined peak and onset temperatures as a function of the Al concentration within Mo-Cr-Ti-Al. The same types of symbols are used in a) and b). Please note that the dotted lines are to guide the eyes.

Compressive stress-strain curves in dependence of temperature were compiled from RT up to $800^{\circ} \mathrm{C}$. The results are depicted in Fig. $6 \mathrm{a} \& \mathrm{~b}$. At RT and $400^{\circ} \mathrm{C}$, MoCrTi-15Al does not exhibit significant plastic deformation. The strain to fracture at $800^{\circ} \mathrm{C}$ is still less than $5 \%$. The alloy exhibits a distinct yield strength plateau over temperature. A similar strength plateau has been reported for the equiatomic MoCrTiAl [18]. Beyond the yield point, strain softening is observed as well as discontinuous plastic deformation. The individual serration events are highlighted by black arrows in the insets of Fig. $6 \mathrm{a} \& \mathrm{~b}$. The serrations in MoCrTi-15Al exhibit a periodic pattern with amplitudes in the range of 20 to $50 \mathrm{MPa}$ (see inset of Fig. 6a) and their characteristics and amplitude are not changing with temperature in the range from 400 to $800^{\circ} \mathrm{C}$. In case of MoCrTi-10Al, similar serrations are observed at 400 and $600^{\circ} \mathrm{C}$. However, at $800^{\circ} \mathrm{C}$ only intermittently serrations at an amplitude of less than $5 \mathrm{MPa}$ are discernible. Similar features in compression have been reported for the B2 ordered NbMoCrTiAl at $400{ }^{\circ} \mathrm{C}[32,38]$ as well as MoNbTaW [39]. The serrations may arise from the pinning and unpinning process of dislocations at APDBs in ordered crystals as it was discussed for B2 ordered Nb-15Al-20V [40]. The alloy likely does not exhibit perfect order due to its non-equimolar composition. Hence, there might be certain contributions to serrations as observed for disordered solid solutions as well. These are discussed in the following paragraph.

MoCrTi-3Al exhibits an ultimate plastic strain of $3 \%$, even at RT as depicted in Fig. 6 b. The strain to fracture at elevated temperatures increases up to $15 \%$ at 400 and $600{ }^{\circ} \mathrm{C}$ and up to $18 \%$ at $800^{\circ} \mathrm{C}$. At temperatures between 400 and $800^{\circ} \mathrm{C}$, the yield strength does not significantly change. Additionally, beyond yielding a discontinuous stress-strain dependence is observed both in MoCrTi-5Al and in MoCrTi-3Al. The amplitude of the serrations is less than $10 \mathrm{MPa}$ and the serrations occur intermittently (see inset of Fig. 6b). Both features, yield strength plateau and serrated plastic flow were preliminarily discussed for dilute solid solutions by Portevin-Le Châtelier (PLC) [41,42]. The PLC effect is explained by the pinning and unpinning of mobile dislocations. Therefore, solute atoms are required to be sufficiently mobile to migrate along moving dislocations. Due to the intrinsic features of HEAs, it is 
complex to assess diffusion-based processes, because each constituent element can be regarded as a solute atom [43].

The yield strength characteristics can be explained by accounting for the respective homologous temperatures $T_{\text {hom }}=T / T_{\mathrm{S}}$. The testing temperatures of RT, 400,600 and $800^{\circ} \mathrm{C}$ correspond $T_{\text {hom }}$ of $0.14,0.32,0.42$ and 0.52 , respectively. In dilute solid solutions, the mobility of screw dislocations is low at low temperatures due to the core structure of screw dislocation inherent to bcc metals and alloys. Thermally activated kink pair formation and propagation determine a significant decrease of the onset of plastic deformation with increasing temperature. In contrast, above $T_{\text {hom }}=0.2$ to 0.4 [25] the mobility of edge and screw dislocations reach the same order of magnitude and the contribution of thermal activation becomes minor. Therefore, a yield strength plateau is observed (note that the experimentally determined shear moduli are rather insensitive to temperature up to $800^{\circ} \mathrm{C}$ as seen for other RHEAs and do not lead to a significantly decreasing strength [10]). For the present alloy, the transition occurs at about $400^{\circ} \mathrm{C}$ corresponding to a homologous temperature of 0.32 . Furthermore, the yield strength plateau extends at least to the highest test temperature of $800^{\circ} \mathrm{C}$ which corresponds to $T_{\text {hom }}=0.52$. This is in accordance with the ascertained transition from the dislocation glide controlled plateau to the creep controlled drop in yield strength at approximately $0.6 \cdot T_{\mathrm{S}}$ in other single-phase RHEAs [44].

The change from an (partially) ordered to a disordered crystal structure between 10 and 5 at $\% \mathrm{Al}$ has a significant impact on the relative ductility and strength in the Mo-Cr-Ti-Al system. The respective change from 15 to 10 at $\% \mathrm{Al}$ (B2 ordered) or from 5 to 3 at $\%$ Al (disordered $\mathrm{A} 2$ ) did not result in noteworthy macroscopic impact. Besides, $T_{\text {hom }}$ slightly decreases for constant, absolute testing temperature due to the increasing absolute $T_{\mathrm{S}}$ through the Al reduction - therefore, an opposite trend regarding ductility was expected which is not the case. The yield strength at the plateau drops through the change from $\mathrm{B} 2$ to $\mathrm{A} 2$ crystal structure by about $375 \mathrm{MPa}$, even though the composition did not vary significantly between MoCrTi-10Al (1350 MPa) and MoCrTi-5Al (975 MPa). This indicates that the critical shear stress for dislocation motion is altered through the change in order. These results lead to the conclusion that the presence of order determines the ductility and yield strength in alloys from the Mo-Cr-Ti-Al system.
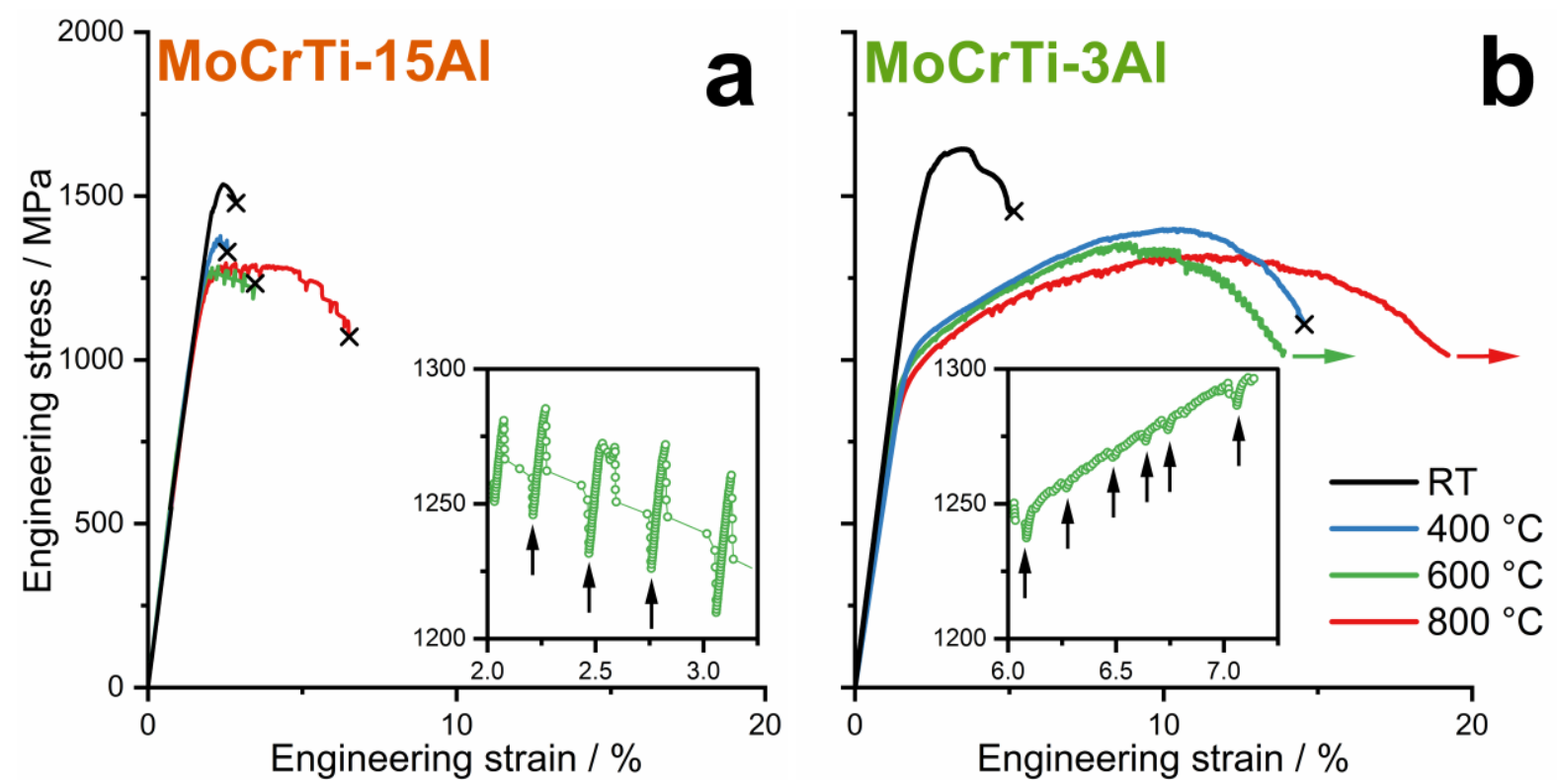

Fig. 6: Stress-strain curves of quasistatic compression tests at different temperatures with an initial strain rate of $10^{-3} \mathrm{~s}^{-1}:$ a) MoCrTi-15Al; b) MoCrTi-3Al. Serrations are highlighted by arrows in the insets in a) and b). The calculated solidus 
temperatures for the investigated alloys are between 1800 and $1900^{\circ} \mathrm{C}$. Lines in the insets are to guide the eyes. Fracture is highlighted by " $\mathrm{x}$ ".

\section{Conclusions}

Guided by thermodynamic calculations, the Al concentration was systematically varied in order to compare single-phase alloys with ordered B2 and disordered A2 crystal structures. Arc melted and subsequent homogenized samples of MoCrTi- $x \mathrm{Al}(x=25,15,10,5,3$ at $\%)$ were investigated.

1. Superlattice Bragg peaks are not observed in MoCrTi-15Al by XRD. In contrast, MoCrTi-10Al exhibits a clear $\{100\}$ Bragg peak. However, TEM-SAD experiments of MoCrTi-15Al unambiguously reveal superlattice spots. Hence, the site occupation within the ordered B2 crystal structure changes by the reduction of Al.

2. High temperature DSC reveals an A2-B2 second-order phase transformation at 965 and $860^{\circ} \mathrm{C}$ for MoCrTi-15Al and MoCrTi-10Al, respectively. The results obtained by DSC confirm the phase transformations predicted by thermodynamic calculations.

3. XRD, TEM-SAD and DSC prove the absence of long-range order in MoCrTi-5Al and MoCrTi$3 \mathrm{Al}$ since neither superlattice diffraction peaks nor $\lambda$-shaped heat signatures are found.

4. The absence of B2 long-range order in MoCrTi-5Al and MoCrTi-3Al leads to higher ultimate plastic strains in compression in the entire temperature range and significantly lower plateau yield strength. The distinct yield strength plateau is observed in conjunction with serrated plastic flow under quasistatic compression at temperatures of $400^{\circ} \mathrm{C}$ and above. In case of alloys with B2 crystal structure, the stress serrations of rather high magnitude might be related to the interaction of dislocations with thermal APDBs.

\section{Acknowledgments}

The financial support by the Deutsche Forschungsgemeinschaft (DFG), grant no. HE 1872/34-1 is gratefully acknowledged. The authors acknowledge the chemical analysis by ICP-OES at the Institute for Applied Materials (IAM-AWP), Karlsruhe Institute of Technology (KIT). Part of this work was performed at the Micro- and Nanoanalytics Facility (MNaF) of the University of Siegen. We also thank A. Srinivasan Tirunilai for proofreading the manuscript and fruitful discussions. Furthermore, we would like to express our gratitude to S. Obert, B.-D. Nguyen, L. Wengenmayer and D. Probst (KIT IAM-WK) for experimental support.

\section{References}

[1] J.-W. Yeh, S.-K. Chen, S.-J. Lin, J.-Y. Gan, T.-S. Chin, T.-T. Shun, C.-H. Tsau et al., Nanostructured High-Entropy Alloys with Multiple Principal Elements: Novel Alloy Design Concepts and Outcomes, Advanced Engineering Materials 6 (2004) 299-303. https://doi.org/10.1002/adem.200300567.

[2] D.B. Miracle, O.N. Senkov, A critical review of high entropy alloys and related concepts, Acta Materialia 122 (2017) 448-511. https://doi.org/10.1016/j.actamat.2016.08.081.

[3] S. Gorsse, D.B. Miracle, O.N. Senkov, Mapping the world of complex concentrated alloys, Acta Materialia 135 (2017) 177-187. https://doi.org/10.1016/j.actamat.2017.06.027.

[4] S. Gorsse, J.-P. Couzinié, D.B. Miracle, From high-entropy alloys to complex concentrated alloys, Comptes Rendus Physique 19 (2018) 721-736. https://doi.org/10.1016/j.crhy.2018.09.004. 
[5] E.P. George, D. Raabe, R.O. Ritchie, High-entropy alloys, Nature Reviews Materials 4 (2019) 515-534. https://doi.org/10.1038/s41578-019-0121-4.

[6] O.N. Senkov, G.B. Wilks, D.B. Miracle, C.P. Chuang, P.K. Liaw, Refractory high-entropy alloys, Intermetallics 18 (2010) 1758-1765. https://doi.org/10.1016/j.intermet.2010.05.014.

[7] C.-Y. Hsu, C.-C. Juan, W.-R. Wang, T.-S. Sheu, J.-W. Yeh, S.-K. Chen, On the superior hot hardness and softening resistance of AlCoCrxFeMo0.5Ni high-entropy alloys, Materials Science and Engineering: A 528 (2011) 3581-3588. https://doi.org/10.1016/j.msea.2011.01.072.

[8] O.N. Senkov, G.B. Wilks, J.M. Scott, D.B. Miracle, Mechanical properties of $\mathrm{Nb} 25 \mathrm{Mo} 25 \mathrm{Ta} 25 \mathrm{~W} 25$ and V20Nb20Mo20Ta20W20 refractory high entropy alloys, Intermetallics 19 (2011) 698-706. https://doi.org/10.1016/j.intermet.2011.01.004.

[9] O.N. Senkov, S.V. Senkova, D.B. Miracle, C. Woodward, Mechanical properties of low-density, refractory multi-principal element alloys of the $\mathrm{Cr}-\mathrm{Nb}-\mathrm{Ti}-\mathrm{V}-\mathrm{Zr}$ system, Materials Science and Engineering: A 565 (2013) 51-62. https://doi.org/10.1016/j.msea.2012.12.018.

[10] O.N. Senkov, C. Woodward, D.B. Miracle, Microstructure and Properties of AluminumContaining Refractory High-Entropy Alloys, JOM 66 (2014) 2030-2042. https://doi.org/10.1007/s11837-014-1066-0.

[11] N.Y. Yurchenko, N.D. Stepanov, S.V. Zherebtsov, M.A. Tikhonovsky, G.A. Salishchev, Structure and mechanical properties of B2 ordered refractory AlNbTiVZrx $(x=0-1.5)$ highentropy alloys, Materials Science and Engineering: A 704 (2017) 82-90. https://doi.org/10.1016/j.msea.2017.08.019.

[12] B.D. Miracle, D.J. Miller, N.O. Senkov, C. Woodward, D.M. Uchic, J. Tiley, Exploration and Development of High Entropy Alloys for Structural Applications, Entropy 16 (2014) 494-525. https://doi.org/10.3390/e16010494.

[13] O.N. Senkov, C.F. Woodward, Microstructure and properties of a refractory NbCrMo0.5Ta0.5TiZr alloy, Materials Science and Engineering: A 529 (2011) 311-320. https://doi.org/10.1016/j.msea.2011.09.033.

[14] F. Körmann, A.V. Ruban, M.H.F. Sluiter, Long-ranged interactions in bcc NbMoTaW highentropy alloys, Materials Research Letters 5 (2017) 35-40. https://doi.org/10.1080/21663831.2016.1198837.

[15] W.P. Huhn, M. Widom, Prediction of A2 to B2 Phase Transition in the High-Entropy Alloy Mo-Nb-Ta-W, JOM 65 (2013) 1772-1779. https://doi.org/10.1007/s11837-013-0772-3.

[16] O.N. Senkov, S.V. Senkova, D.M. Dimiduk, C. Woodward, D.B. Miracle, Oxidation behavior of a refractory NbCrMo0.5Ta0.5TiZr alloy, Journal of Materials Science 47 (2012) 6522-6534. https://doi.org/10.1007/s10853-012-6582-0.

[17] O.N. Senkov, J.M. Scott, S.V. Senkova, F. Meisenkothen, D.B. Miracle, C.F. Woodward, Microstructure and elevated temperature properties of a refractory TaNbHfZrTi alloy, Journal of Materials Science 47 (2012) 4062-4074. https://doi.org/10.1007/s10853-012-6260-2.

[18] H. Chen, A. Kauffmann, S. Laube, I.-C. Choi, R. Schwaiger, Y. Huang, K. Lichtenberg et al., Contribution of Lattice Distortion to Solid Solution Strengthening in a Series of Refractory High 

https://doi.org/10.1007/s11661-017-4386-1.

[19] O.N. Senkov, D.B. Miracle, K.J. Chaput, J.-P. Couzinie, Development and exploration of refractory high entropy alloys-A review, Journal of Materials Research 33 (2018) 3092-3128. https://doi.org/10.1557/jmr.2018.153.

[20] O.N. Senkov, S.V. Senkova, C. Woodward, Effect of aluminum on the microstructure and properties of two refractory high-entropy alloys, Acta Materialia 68 (2014) 214-228. https://doi.org/10.1016/j.actamat.2014.01.029.

[21] N.I. Medvedeva, Y.N. Gornostyrev, D.L. Novikov, O.N. Mryasov, A.J. Freeman, Ternary site preference energies, size misfits and solid solution hardening in $\mathrm{NiAl}$ and $\mathrm{FeAl}$, Acta Materialia 46 (1998) 3433-3442. https://doi.org/10.1016/S1359-6454(98)00042-1.

[22] M. Ogura, T. Fukushima, R. Zeller, P.H. Dederichs, Structure of the high-entropy alloy AlxCrFeCoNi: fcc versus bcc, Journal of Alloys and Compounds 715 (2017) 454-459. https://doi.org/10.1016/j.jallcom.2017.04.318.

[23] A. Seeger, On the theory of the low-temperature internal friction peak observed in metals, The Philosophical Magazine: A Journal of Theoretical Experimental and Applied Physics 1 (1956) 651-662. https://doi.org/10.1080/14786435608244000.

[24] T. Suzuki, H. Koizumi, H.O.K. Kirchner, Plastic flow stress of b.c.c. transition metals and the Peierls potential, Acta Metallurgica et Materialia 43 (1995) 2177-2187. https://doi.org/10.1016/0956-7151(94)00451-X.

[25] A. Seeger, B. Sestak, Glide and Work-hardening in bcc Metals and Alloys (I-III), Zeitschrift für Metallkunde (1978) 195-202.

[26] J.-P. Couzinié, G. Dirras, Body-centered cubic high-entropy alloys: From processing to underlying deformation mechanisms, Materials Characterization 147 (2019) 533-544. https://doi.org/10.1016/j.matchar.2018.07.015.

[27] M. Feuerbacher, Dislocations and deformation microstructure in a B2-ordered A128Co20Cr11Fe15Ni26 high-entropy alloy, Scientific Reports 6 (2016). https://doi.org/10.1038/srep29700.

[28] S.I. Rao, C. Varvenne, C. Woodward, T.A. Parthasarathy, D. Miracle, O.N. Senkov, W.A. Curtin, Atomistic simulations of dislocations in a model BCC multicomponent concentrated solid solution alloy, Acta Materialia 125 (2017) 311-320. https://doi.org/10.1016/j.actamat.2016.12.011.

[29] F. Maresca, W.A. Curtin, Mechanistic origin of high retained strength in refractory BCC high entropy alloys up to 1900K, arXiv preprint (2019).

[30] J.R. Rice, R. Thomson, Ductile versus brittle behaviour of crystals, The Philosophical Magazine: A Journal of Theoretical Experimental and Applied Physics 29 (1974) 73-97. https://doi.org/10.1080/14786437408213555.

[31] P. Gumbsch, J. Riedle, A. Hartmaier, H.F. Fischmeister, Controlling factors for the brittle-toductile transition in tungsten single crystals, Science 282 (1998) 1293-1295. https://doi.org/10.1126/science.282.5392.1293. 
[32] H. Chen, A. Kauffmann, S. Seils, T. Boll, C.H. Liebscher, I. Harding, K.S. Kumar et al., Crystallographic ordering in a series of Al-containing refractory high entropy alloys $\mathrm{Ta}-\mathrm{Nb}-\mathrm{Mo}-$ Cr-Ti-Al, Acta Materialia 176 (2019) 123-133. https://doi.org/10.1016/j.actamat.2019.07.001.

[33] F. Müller, B. Gorr, H.-J. Christ, J. Müller, B. Butz, H. Chen, A. Kauffmann et al., On the oxidation mechanism of refractory high entropy alloys, Corrosion Science 159 (2019) 108161. https://doi.org/10.1016/j.corsci.2019.108161.

[34] J.B. Nelson, D.P. Riley, An experimental investigation of extrapolation methods in the derivation of accurate unit-cell dimensions of crystals, Proceedings of the Physical Society 57 (1945) 160-177. https://doi.org/10.1088/0959-5309/57/3/302.

[35] R.W. Balluffi, L.L. Seigle, Growth of voids in metals during diffusion and creep, Acta Metallurgica 5 (1957) 449-454. https://doi.org/10.1016/0001-6160(57)90063-9.

[36] R. Santamarta, E. Cesari, J. Font, J. Muntasell, J. Pons, J. Dutkiewicz, Effect of atomic order on the martensitic transformation of Ni-Fe-Ga alloys, Scripta Materialia 54 (2006) 1985-1989. https://doi.org/10.1016/j.scriptamat.2006.03.018.

[37] D.A. Porter, K.E. Easterling, M. Sherif, Phase Transformations in Metals and Alloys, 3rd ed., CRC Press, Hoboken, 2009.

[38] H. Chen, A. Kauffmann, B. Gorr, D. Schliephake, C. Seemüller, J.N. Wagner, H.-J. Christ et al., Microstructure and mechanical properties at elevated temperatures of a new Al-containing refractory high-entropy alloy Nb-Mo-Cr-Ti-Al, Journal of Alloys and Compounds 661 (2016) 206-215. https://doi.org/10.1016/j.jallcom.2015.11.050.

[39] J. Antonaglia, X. Xie, Z. Tang, C.-W. Tsai, J.W. Qiao, Y. Zhang, M.O. Laktionova et al., Temperature Effects on Deformation and Serration Behavior of High-Entropy Alloys (HEAs), JOM 66 (2014) 2002-2008. https://doi.org/10.1007/s11837-014-1130-9.

[40] T.S. Rong, Serrated yielding in the B2-ordered Nb-15Al-20V alloy, Intermetallics 11 (2003) 151-155. https://doi.org/10.1016/S0966-9795(02)00196-6.

[41] P. Rodriguez, Serrated plastic flow, Bulletin of Materials Science 6 (1984) 653-663. https://doi.org/10.1007/BF02743993.

[42] A. Portevin, F. Le Chatelier, Sur un phénomène observé lors de l'essai de traction d'alliages en cours de transformation, Comptes Rendus de l'Académie des Sciences Paris 176 (1923) 507510 .

[43] C. Varvenne, G.P.M. Leyson, M. Ghazisaeidi, W.A. Curtin, Solute strengthening in random alloys, Acta Materialia 124 (2017) 660-683. https://doi.org/10.1016/j.actamat.2016.09.046.

[44] O.N. Senkov, S. Gorsse, D.B. Miracle, High temperature strength of refractory complex concentrated alloys, Acta Materialia 175 (2019) 394-405. https://doi.org/10.1016/j.actamat.2019.06.032. 